\title{
A fractional-order model on the dynamic mechanical behavior of magnetorheological elastomers
}

\author{
Zhu Guanghong ${ }^{\mathrm{a} *}$, Xiong Yeping ${ }^{\mathrm{b}}$, Li Zigang ${ }^{\mathrm{a}}$, Li Ming ${ }^{\mathrm{a}}$, Bai Xianxu ${ }^{\mathrm{c}}$ \\ aDepartment of Mechanics, Xi' an University of Science and Technology, Xi'an 710054, P. R. China \\ ${ }^{b}$ Faculty of Engineering and the Physical Sciences, University of Southampton, Southampton SO16 7QF, UK \\ ${ }^{c}$ Laboratory for Adaptive Structures and Intelligent Systems (LASIS), Department of Vehicle Engineering, Hefei \\ University of Technology, Hefei 230009, P. R. China
}

\begin{abstract}
In order to describe the dynamic behavior of magnetorheological elastomers (MREs) for the realization of vibration control, this study proposes a constitutive model containing a fractional element and nonlinear springs attributed to the viscoelastic and the rheological properties, respectively. The viscoelastic behavior in various magnetic fields was studied experimentally to develop this fractional-order nonlinear model, and the model parameters were identified through experimental data fitting of dynamic modulus in frequency domain. The model predictions were subsequently obtained with the predictor-corrector approach to validate the proposed model by comparing with the experimental results on the stress-strain hysteresis. In addition, the efficiency of the proposed model of MRE was also evaluated by comparing the numerical solution with the results of the revised Bouc-Wen model.
\end{abstract}

Keywords: fractional-order model, nonlinear model, magnetorheological elastomers, dynamic mechanical analysis, hysteresis

\section{Introduction}

Magnetorheological elastomers (MREs) are a class of composite materials that consist of magnetizable particles suspended in an elastomer matrix with low permeability. Anisotropic MREs have a directed particle orientation as a result of the presence of a magnetic field during the solidification, and isotropic MREs can be characterized with a random distribution of magnetic particles attributed to the curing progress without any magnetic fields ${ }^{[1,2]}$. However, with the application of an external field even isotropic MREs become anisotropic materials ${ }^{[3]}$.

There has been increasing research on MRE for reduction of undesired vibrations $^{[4,5]}$. As a combination of the versatility of active control and the reliability of passive control, the semi-active (adaptive-passive) vibration control devises have attracted considerable intention over the past decades and the increasing effort has been 
devoted to the possibility of employing smart materials for semi-active vibration control ${ }^{[6,7]}$. Among these suited smart materials, MRE is promising in practice due to the controllable rheological property ${ }^{[3,8,9]}$, which has been applied in varies fields, such as the automotive industry ${ }^{[10,11]}$ and civil engineering ${ }^{[12]}$.

Because MREs have been applied broadly for semi-active control, and the dynamic mechanical properties can be influenced by not only the components and the fabrication but also by the in-service condition ${ }^{[13,14,15]}$; how to develop a quality constitutive model of MRE is a foremost problem for the further application on semi-active control. Since Jolly et al. developed a dipole model which is basically one-dimensional and quasistatic to describe the field-induced modulus of MRE, chain models and column models have been proposed to deduce the field-induced shear modulus and the optimum particle volume fraction ${ }^{[16,17,18]}$. Their research has focused on the dependence of the dynamic modulus on magnetic fields, and besides that as a viscoelastic material, the dynamic mechanical properties are also affected by temperature ${ }^{[19,20]}$, frequency and deformation. Based upon the Kelvin-Voigt model and the Maxwell model for viscoelastic materials, three-parameter models and four-parameter models were respectively developed to describe the creep and the relaxation phenomena ${ }^{[21,22]}$. As the deformation of viscoelastic materials is dependent on time, the classical viscoelastic model cannot work well. Bouc-Wen model and Ramberg-Osgood model were employed in parallel with viscoelastic model to describe the hysteresis behavior ${ }^{[23,24]}$; and the disadvantage of classical viscoelastic model can be also remedied with fractional elements, in which the stress-strain relation is expressed by fractional derivatives ${ }^{[25,26,27]}$. Recently, an optimal fractional rheological model was constructed for MREs, and the interaction between the viscoelastic properties and the magnetic field was analysed in terms of fractional element order parameters ${ }^{[28,29]}$. Such constitutive models can be further developed by integrating the MR effect ${ }^{[30,31,32]}$. The research on constitutive models of MREs has made progress, however, as an essential step to achieve the great potential of MRE for vibration control, mathematical models with a great efficiency to describe the dynamic mechanical behavior deserve more effort.

In this study, based upon the experimental results of mechanical property characterization, a fractional-order nonlinear model is developed to describe the dynamic mechanical behavior of MREs. The experimental data of dynamic modulus in shear mode is obtained by performing the (dynamic mechanical analysis) DMA tests, and a constitutive model of MRE is developed with the incorporation of the rheological 
behavior and the viscoelastic behavior. By optimizing errors with the genetic algorithm, the model parameters are identified through data fitting of dynamic modulus in frequency domain. The numerical solutions of the constitutive equation are obtained through the predictor-corrector approach, and the model predictions are compared with the experimental results on the stress-strain hysteresis. Eventually, the accuracy of this proposed model is evaluated by comparing with both the experimental data and the result of the revised Bouc-Wen model ${ }^{[31]}$ in time domain.

\section{DMA experiments}

The anisotropic MRE samples in this study were comprised of the micron-sized iron particles (Sigma-Aldrich, US) and the silicone rubber (Wacker Chemie AG, Germany). The manufacturing procedure can be illustrated by three steps: firstly, there were two components Elastosil A and Elastosil B mixed for silicone rubber with a ratio of 10:1 by volume, and the carbonyl iron powders sized up to $9 \mu \mathrm{m}$ were added to the mixture with a volume concentration of $30 \%$, which is considered to be able to generate an obvious MR effect ${ }^{[16,17,18]}$; secondly, the mixture was blended thoroughly and then placed in a vacuum chamber for 20 minutes for removing air bubbles trapped inside; finally, the mixture was put into square aluminum moulds $21.8 \times 21.8 \times 6.5 \mathrm{~mm}^{3}$ and cured for 16 hours at room temperature for solidification with a magnetic field, which was of $290 \mathrm{mT}$ with air-dielectric and was produced by cylindrical grade N42 neodymium permanent magnets (E-magnets, UK).

According to the BS ISO 4664-1:2011 for shear modes, the DMA tests of MRE samples were performed using Instron Electropuls E1000, which is able to apply and control the harmonic shear strain, as shown in Figure 1. The influence of magnetic field on the dynamic properties was also obtained by the use of cylindrical grade N42 neodymium permanent magnets, meanwhile the force and the displacement were tracked by Instron Electropuls E1000 during tests. The dynamic mechanical behavior of MRE were measured with varying frequencies from $1 \mathrm{~Hz}$ to $50 \mathrm{~Hz}$, strain amplitudes from $1 \%$ to $5 \%$, and magnetic fields from 0 to $500 \mathrm{mT}$. And all the sets of tests were carried out with three independent pairs of samples at room temperature (about $25^{\circ} \mathrm{C}$ ). 


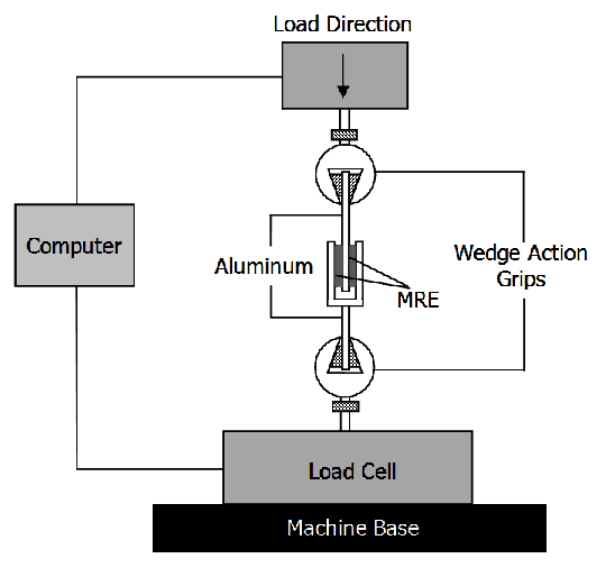

(a)

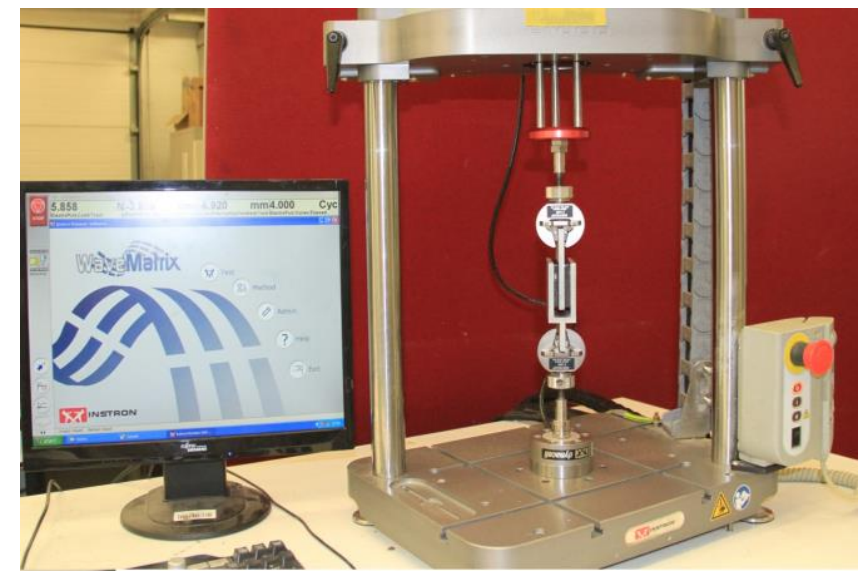

(b)

Figure 1 Experimental setup for DMA tests of MRE in shear mode (a) schematic and (b) photograph.

When the applied loads are sinusoidal, the strain of MRE materials has the following form:

$$
(t)={ }_{0} \sin t
$$

where $\omega=2 \pi f ; \varepsilon_{0}, \omega$ and $f$ are the strain amplitude, the angular frequency and the frequency, respectively. Because of viscoelasticity the response will not be instantaneous, the resulting stress will lag behind the input load by an angle $\varphi$ called loss angle whose range is $0<\varphi<90^{\circ}$. For viscoelastic materials, some of the deformation energy can be stored and recovered, whilst the remainder is dissipated as heat during each cycle. The storage modulus $M^{\prime}$ contributes to the material stiffness and represents the ability of storing the energy due to deformation; and the loss modulus $M^{\prime \prime}$ indicates the ability of viscoelastic material to dissipate the energy of deformation. They can be defined as:

$$
\sigma=\varepsilon_{0}\left(M^{\prime} \sin \omega t+M^{\prime \prime} \cos \omega t\right)
$$


where $\sigma$ denotes the stress; the modulus $M$ can be either the Young's modulus $E$ and the shear modulus $G$. Because the dependence of the in-phase and out-of-phase stress on the strain is conveniently expressed by a complex modulus, the modulus $M^{*}$ is usually presented as a complex quantity:

$$
M^{*}=M^{\prime}+i M^{\prime \prime}
$$

The ratio between the loss modulus $M^{\prime \prime}$ and storage modulus $M^{\prime}$ is introduced as a widely used term for viscoelastic materials:

$$
\tan \varphi=\frac{M^{\prime \prime}}{M^{\prime}}
$$

where $\tan \varphi$ is called the loss factor using for describing the efficiency of damping caused by the viscoelastic material.

According to the DMA directions in this study, the complex modulus $M^{*}$ can be calculated as the ratio of the stress range to the strain range. The loss angle $\varphi$ is relevant to the energy dissipation within an oscillatory cycle, which is the area enclosed by the hysteresis loop and can be calculated by the numerical integration. The storage modulus $M^{\prime}$, loss modulus $M^{\prime \prime}$ and loss angle $\varphi$ can be defined as:

$$
\begin{aligned}
M^{\prime} & =M \cos \\
M^{\prime \prime} & =M \sin \\
& =\arcsin \frac{E_{\text {loop }}}{A_{\text {strain }} A_{\text {stress }}}
\end{aligned}
$$

where $A_{\text {strain }}$ and $A_{\text {stress }}$ denote the strain amplitude and the stress amplitude, respectively; and $E_{\text {loop }}$ indicates the energy enclosed by the hysteresis loop where the numerical integration can reduce the effects of measurement noise and take into account waveform distortion.

\section{Constitutive model of MRE}

The dynamic mechanical behavior of MRE under harmonic loads can be described by the constitutive equation of viscoelastic materials. Evidently, the viscoelastic phenomenon can be observed in typical situations, such as sinusoidal dynamic loading, creep and relaxation. With a spring and a viscous dashpot arranged in parallel, simple Voigt elements are mostly used to describe the creep, where deformation increases along time at imposed stress; while the relaxation where stress changes along time at a 
constant deformation can be depicted by simple Maxwell elements, which consists of a spring and a viscous dashpot arranged in series.

Based on the two classic models above, a fractional element is introduced to describe the characteristics of viscoelastic materials, which perform between viscous and elastic behaviors ${ }^{[33]}$. The relationship between stress and strain can be expressed in the following form,

$$
{ }_{v}=G_{10} D[(t)] 0<<1
$$

where $G_{l}, \tau_{v}$ and $\gamma_{v}$ are the shear modulus, the shear stress and the shear strain of the fractional element, respectively; $\tau_{0}$ denotes the relaxation time constant; $D$ denotes the derivative operator; $\alpha$ is a fractional-order. In the extreme cases of $\alpha=1$ and $\alpha=0$, the fractional element turns into a Newton dashpot and a linear spring, respectively.

As the external magnetic field applies, the interaction between magnetized particles within MREs will generate a field-induced modulus. Therefore, the magnetic fieldinduced modulus can be described by a nonlinear spring in parallel with a viscoelastic model. Since the first dipole model, chain models and column models have been developed to investigate the field-induced shear modulus and predicted the optimum particle volume fraction. The field-induced shear modulus is commonly expressed as a quadratic function of the magnetic flux density until the saturation of magnetization $^{[16,17,18]}$, and can be calculated with the following expression ${ }^{[18]}$ :

$$
G_{m}=\left(\begin{array}{ll}
P & V
\end{array}\right) H_{0}^{2} \sin \cos
$$

where $H_{0}$ is the applied magnetic field intensity; $\mu_{\mathrm{P}}$ and $\mu_{\mathrm{v}}$ are the permeabilities of the block parallel and perpendicular to the column axes, respectively. In this equation, it is obvious that the magnetic field-induced modulus $G_{m}$ is a nonlinear function of not only the magnetic field but also the strain.

In the constitutive model of MRE, as shown in Figure 2, the Zener rheological model is improved with the substitution of classical springs and dashpots. A spring and a fractional element are arranged in series to describe the dominant elasticity and the viscoelasticity. Because the damping characteristic of MREs can be hardly influenced by field-induced modulus, another spring is arranged in parallel in the constitutive model to describe the field-induced modulus. As illustrated preciously the stiffness of the two springs herein depends on magnetic field, frequency and deformation, and the field-induced shear stress can be expressed as: 


$$
{ }_{m}=G_{m}(t)
$$

where $\gamma$ is the total shear strain of this constitutive model.

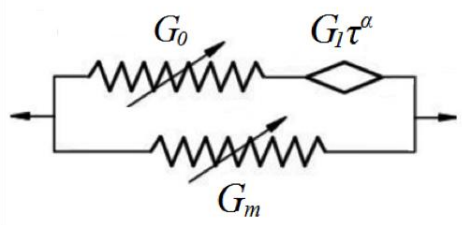

Figure 2 Constitutive model of MRE.

Subsequently, the shear strain and the shear stress in the constitutive model can be expressed as:

$$
\begin{aligned}
& (t)={ }_{e}(t)+{ }_{v}(t) \\
& ={ }_{v}+{ }_{m}
\end{aligned}
$$

where $\gamma_{e}$ is the shear strain of the linear spring.

The relationship between the shear stress and the shear strain in the constitutive model can be derived in time domain as:

$$
(t)=\frac{G_{0} G_{m}+\left(G_{0}+G_{m}\right) G_{10} D}{G_{0}+G_{10} D}(t)
$$

With the Taylor series expansions of sine and cosine, the magnetic field-induced modulus $G_{m}$ can be rewritten from Equation (9).

$$
G_{m}=\left(\begin{array}{ll}
P & V
\end{array}\right) H_{0}^{2}[1+()]
$$

When the shear strain $\gamma$ is sinusoidal and smaller than $6 \%$, the influence of infinitesimal of higher order on the magnetic field-induced modulus $G_{m}$ in Equation (14) will be very limited in short periods. Because the vibration periods of sinusoidal cyclic loads in this study are not more than 1 second, it is assumed that the MR effect is a mean performance during a period with definite vibration amplitudes. Therefore, considering in steady-state magnetic fields the MR effect varies with the vibration amplitude, the magnetic field-induced modulus $G_{m}$ can be simplified as a function of the magnetic field and the strain amplitude. The response of MREs to sinusoidal cyclic loads can be also studied by converting viscoelastic problems into elastic problems with transform methods.

$$
()=\frac{G_{0} G_{m}+\left(G_{0}+G_{m}\right) G_{10}(i)}{G_{0}+G_{10}(i)}()
$$


In general, a dynamic shear modulus $G^{*}$ can be defined as a complex function of the frequency.

$$
G()=\frac{()}{()}=G^{\prime}()+i G^{\prime \prime}()
$$

Applying the Fourier transform and substituting $i^{\alpha}=\cos (\alpha \pi / 2)+i \sin (\alpha \pi / 2)$ into Equation (16), the storage modulus (real component) $G^{\prime}$ can be readily found in the frequency domain:

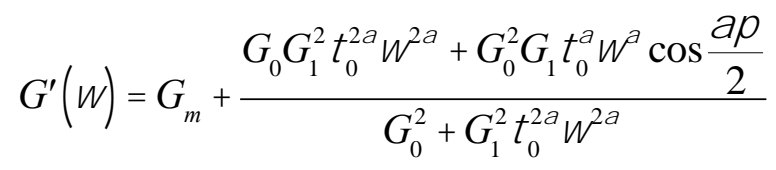

The loss modulus (imaginary component) $G^{\prime \prime}$ can be also derived:

$$
G^{\prime \prime}()=\frac{G_{0}^{2} G_{1} \quad 0 \quad \sin \frac{}{2}}{G_{0}^{2}+G_{1}^{2} 0^{2}}
$$

\section{Parameter identification}

The hysteresis loops and dynamic moduli of MRE have been measured by performing DMA tests of samples within small strain range in shear mode. Figure 3 shows the deformation, frequency and magnetic field dependent hysteresis loops of MRE with frequency of $10 \mathrm{~Hz}$, magnetic flux density of 0 and strain amplitude of $1 \%$. The hysteresis loop is approximately elliptical in shape, and the slop of the main axis of hysteresis loop indicates the dynamic modulus of the MRE sample. It is obvious that the dynamic modulus is reduced by the increasing strain amplitude, and the dynamic modulus exhibits increases as the frequency or the magnetic flux density increases.

The experimental data of storage and loss moduli can be obtained by data processing with Equations (6)-(8), and the dynamic moduli can be also predicted by calculating with Equations (18) and (19). With the use of genetic algorithm (GA) in MATLAB for optimization, the model parameters $G_{0}, G_{l}, G_{m}, \tau_{0}$ and $\alpha$ can be identified by minimizing the sum of square of error between the experimental data and fitted results of storage modulus and loss modulus. 


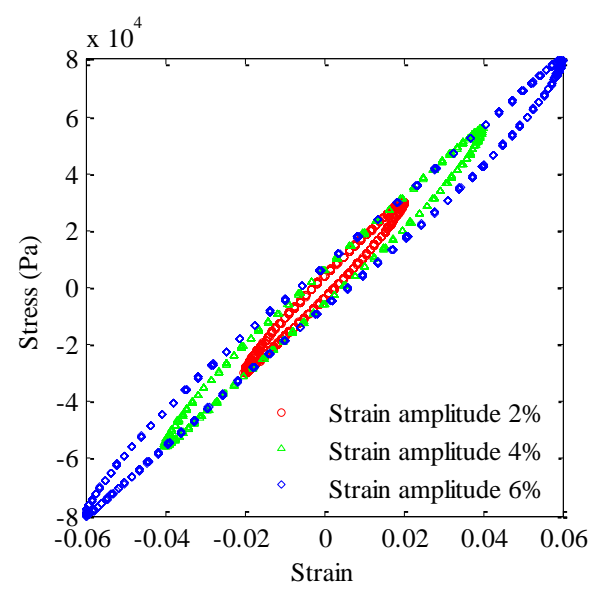

(a)

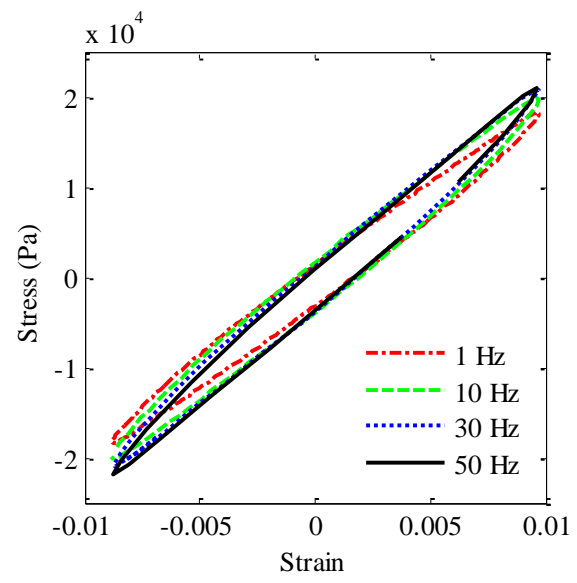

(b)

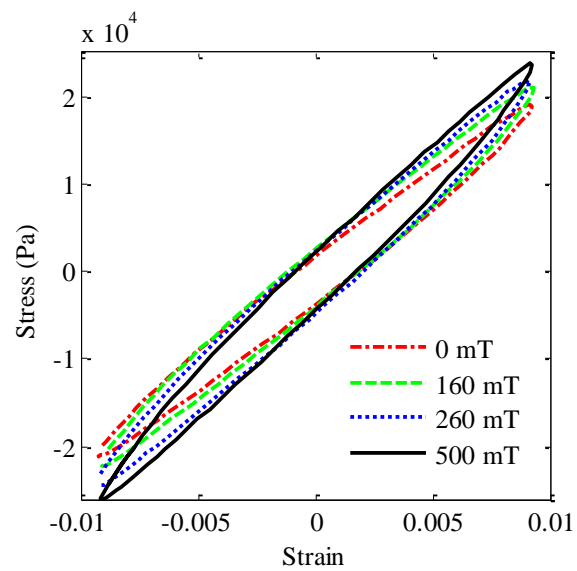

(c)

Figure 3 Dependence of hysteresis loop on (a) strain amplitude, (b) frequency and (c) magnetic field.

The fitting results can be evaluated by measuring the goodness-of-fit. The standard error of deviation $\left(S_{\mathrm{y}}{ }^{2}\right)$, the standard error of estimation $\left(S_{\mathrm{e}}^{2}\right)$ and the coefficient of determination $\left(R^{2}\right)$ are expressed respectively in the following forms ${ }^{[34]}$,

$$
S_{y}^{2}=\frac{\left(y_{\exp } \bar{y}_{\exp }\right)^{2}}{n 1}
$$




$$
\begin{aligned}
& S_{e}^{2}=\frac{\left(y_{\exp } y_{f i t}\right)^{2}}{n} \\
& R^{2}=1 \frac{n}{n} \frac{S_{e}^{2}}{S_{y}^{2}}
\end{aligned}
$$

where $n$ is the number of data samples; $y_{\exp }$ and $y_{f i t}$ are the experimental data and fitted results of modulus, respectively; $y_{\exp }$ denotes the average of experimental data. The data samples $(y)$ can be both storage modulus $G^{\prime}$ and loss modulus $G^{\prime \prime}$.

From Figure 4, it can be observed that the storage modulus and loss modulus of MRE decrease with the strain amplitude, and increase with the magnetic flux density until the saturation of magnetization and remain constant afterwards. As for the dependence on frequency, the storage modulus increases as the frequency increases, and the dependence of loss modulus on frequency is determined by the matrix material, that means the shape of curve is inherited from the matrix material.

In Figure 4, an excellent agreement can be observed between the fitted results and the experimental data in the frequency domain. The model parameters $G_{0}, G_{1}, G_{m}, \tau_{0}$ and $\alpha$ can be identified simultaneously by the experimental data of dynamic moduli in frequency domain, and the results are listed in Table 1. Comparing with the storage modulus $G^{\prime}$, the curve of loss modulus $G^{\prime \prime}$ is more complex and the value is smaller, so the initial identification is carried out with the experimental data of loss modulus $G^{\prime \prime}$. By adjusting the obtained model parameters $G_{0}, G_{l}, \tau_{0}$ and $\alpha$; the parameter $G_{m}$ can be further identified with the experimental data of storage modulus $G^{\prime}$. As a result, a set of model parameters $G_{0}, G_{l}, G_{m}, \tau_{0}$ and $\alpha$ can be identified for both storage $G^{\prime}$ modulus and loss modulus $G^{\prime \prime}$ with a certain amplitude in steady-state magnetic fields. These model parameters can be expressed as functions of magnetic field and strain amplitude, which is also why we choose nonlinear springs in this constitutive model. The coefficient of determination $R^{2}$ and the standard error ratio $S_{\mathrm{e}} / S_{\mathrm{y}}$ can be calculated based on Equations (19)-(21), and the results are listed in Table 2 where the quality of curve fitting is illustrated in detail. Because the values of the two dynamic muduli differ by an order of magnitude, the standard errors of estimation $\left(S_{\mathrm{e}}^{2}\right)$ will also be very different, but the standard error ratio $S_{\mathrm{e}} / S_{\mathrm{y}}$ can not be affected in dimensionless form. According to the criteria when the coefficient of determination $R^{2}>0.90$ and the standard error ratio $S_{\mathrm{e}} / S_{\mathrm{y}}<0.35$, the goodness-of-fit can be accepted to be excellent. 
Table 1 The results of parameter identification.

\begin{tabular}{|c|c|c|c|c|c|c|}
\hline $\begin{array}{l}\text { Magnetic } \\
\text { intensity }\end{array}$ & $\begin{array}{l}\text { Strain } \\
\text { amplitude }\end{array}$ & $G_{0}$ & $G_{l}$ & $G_{m}$ & $\tau_{0}$ & $\alpha$ \\
\hline \multirow{3}{*}{$0 \mathrm{mT}$} & $1 \%$ & 1.4048 & 1.3884 & 0 & 1.6671 & 0.3416 \\
\hline & $3 \%$ & 1.3161 & 1.2692 & 0 & 1.4872 & 0.4482 \\
\hline & $5 \%$ & 1.1354 & 1.1528 & 0 & 1.5291 & 0.3089 \\
\hline \multirow{3}{*}{$160 \mathrm{mT}$} & $1 \%$ & 1.5804 & 1.4774 & 1.1131 & 1.5577 & 0.3222 \\
\hline & $3 \%$ & 0.9042 & 0.8546 & 1.0549 & 1.5947 & 0.3796 \\
\hline & $5 \%$ & 0.8243 & 0.7984 & 1.3559 & 0.9871 & 0.4169 \\
\hline \multirow{3}{*}{$260 \mathrm{mT}$} & $1 \%$ & 1.6615 & 1.5959 & 1.6441 & 1.5808 & 0.3129 \\
\hline & $3 \%$ & 1.0759 & 1.2471 & 1.4177 & 1.5369 & 0.3483 \\
\hline & $5 \%$ & 0.8817 & 0.8257 & 1.4802 & 1.2148 & 0.3857 \\
\hline \multirow{3}{*}{$450 \mathrm{mT}$} & $1 \%$ & 1.7916 & 1.7747 & 1.9306 & 1.5184 & 0.4009 \\
\hline & $3 \%$ & 1.1523 & 1.4280 & 1.6254 & 1.9595 & 0.3619 \\
\hline & $5 \%$ & 0.9913 & 1.0391 & 1.5971 & 1.6490 & 0.3388 \\
\hline \multirow{3}{*}{$500 \mathrm{mT}$} & $1 \%$ & 1.8656 & 1.7994 & 1.9820 & 1.4458 & 0.3054 \\
\hline & $3 \%$ & 1.2721 & 1.0461 & 1.7264 & 2.3952 & 0.3409 \\
\hline & $5 \%$ & 1.1167 & 1.1100 & 1.6488 & 1.9202 & 0.3174 \\
\hline
\end{tabular}

Table 2 The goodness of fit.

\begin{tabular}{|c|c|c|c|c|c|c|c|}
\hline \multirow{2}{*}{\multicolumn{2}{|c|}{$\begin{array}{l}\text { Strain amplitude }(\%) \\
\text { Dynamic moduli }\end{array}$}} & \multirow{2}{*}{$\begin{array}{l}1 \\
G^{\prime}\end{array}$} & \multirow{3}{*}{$\begin{array}{l}G^{\prime \prime} \\
0.1791\end{array}$} & \multicolumn{2}{|l|}{3} & \multicolumn{2}{|l|}{5} \\
\hline & & & & \multirow{2}{*}{$\frac{G^{\prime}}{0.0387}$} & \multirow{2}{*}{$\begin{array}{l}G^{\prime \prime} \\
0.0768\end{array}$} & \multirow{2}{*}{$\frac{G^{\prime}}{0.0185}$} & \multirow{2}{*}{$\begin{array}{l}G^{\prime \prime} \\
0.1367\end{array}$} \\
\hline $0 \mathrm{mT}$ & $S_{\mathrm{e}} / S_{\mathrm{y}}$ & 0.0547 & & & & & \\
\hline & $R^{2}$ & 0.9982 & 0.9825 & 0.9991 & 0.9968 & 0.9974 & 0.9898 \\
\hline \multirow[t]{2}{*}{$160 \mathrm{mT}$} & $S_{\mathrm{e}} / S_{\mathrm{y}}$ & 0.0728 & 0.1158 & 0.0624 & 0.0017 & 0.0316 & 0.1578 \\
\hline & $R^{2}$ & & & & & 0.9994 & 0.9864 \\
\hline \multirow[t]{2}{*}{$260 \mathrm{mT}$} & $S_{\mathrm{e}} / S_{\mathrm{y}}$ & 0.0860 & 0.1326 & 0.0316 & 0.0332 & 0.0854 & 0.2054 \\
\hline & $R^{2}$ & 0.9956 & 0.9904 & 0.9994 & 0.9994 & 0.9956 & 0.9770 \\
\hline \multirow[t]{2}{*}{$450 \mathrm{mT}$} & $S_{\mathrm{e}} / S_{\mathrm{y}}$ & 0.0574 & 0.1449 & 0.1416 & 0.1077 & 0.0916 & 0.1500 \\
\hline & $R^{2}$ & 0.9980 & 0.9886 & 0.9929 & 0.9937 & 0.9949 & 0.9877 \\
\hline \multirow[t]{2}{*}{$500 \mathrm{mT}$} & $S_{\mathrm{e}} / S_{\mathrm{y}}$ & 0.0728 & 0.0787 & 0.0479 & 0.0911 & 0.0282 & 0.0842 \\
\hline & $R^{2}$ & 0.9968 & 0.9966 & 0.9986 & 0.9955 & 0.9995 & 0.9961 \\
\hline
\end{tabular}




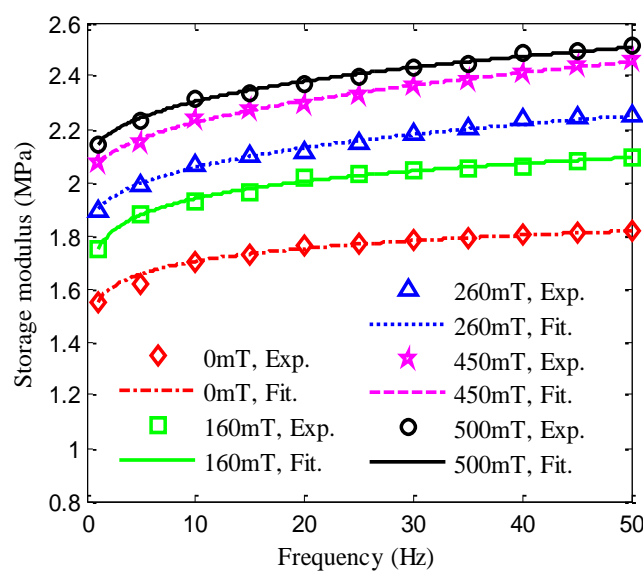

(a)

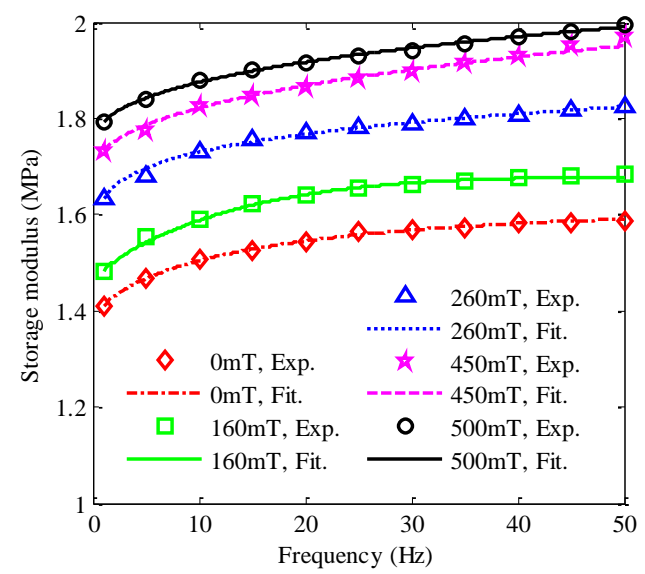

(c)

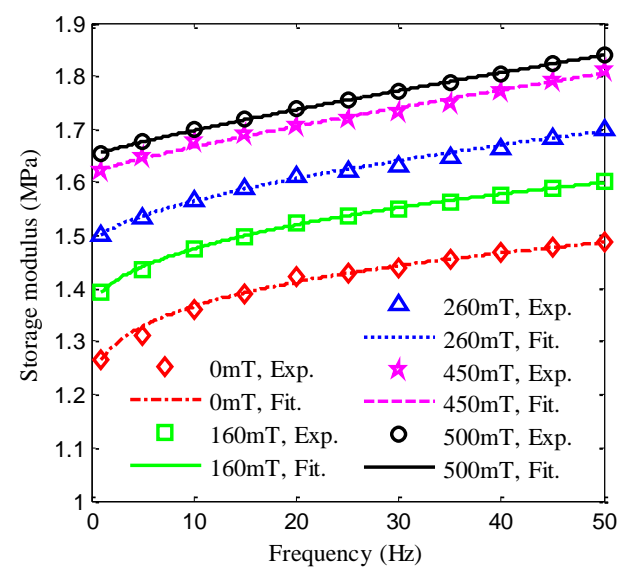

(e)

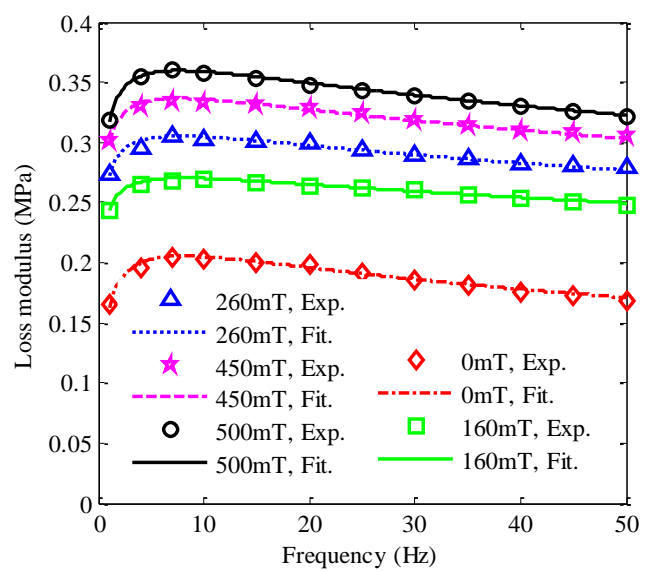

(b)

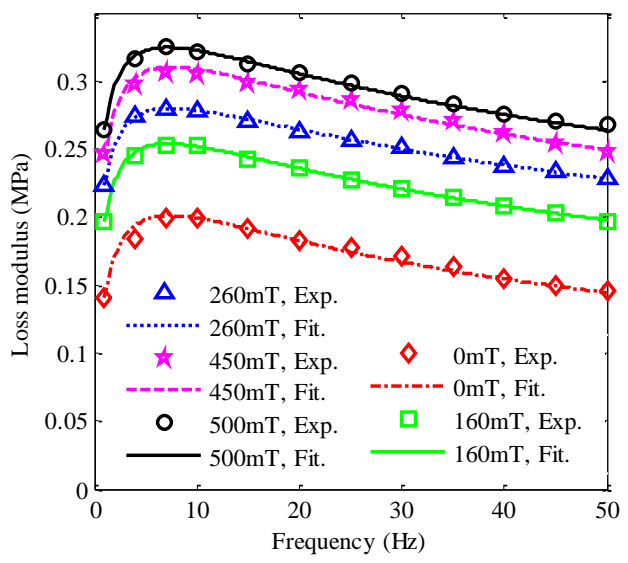

(d)

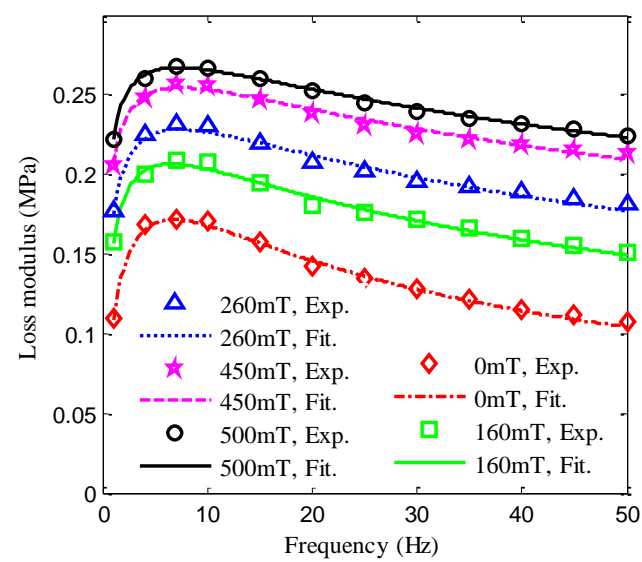

(f)

Figure 4 Comparison between experimental data and fitted results of storage modulus with strain amplitude of (a) 1\%, (c) 3\%, (e) 5\%; and loss modulus with strain amplitude of (b) $1 \%$, (d) $3 \%$, (f) $5 \%$.

\section{Numerical solution and validation}

When we look at a differential equation of fractional-order, equipped with suitable initial conditions, such as 


$$
\begin{aligned}
& D x=f(t, x) \\
& x^{k}(0)=x_{0}^{(k)}
\end{aligned}
$$

where $\alpha>0$ and $[\alpha]$ is the value $\alpha$ rounded up to the nearest integer. There is a close connection between the types of the initial condition and the fractional derivative. The initial conditions for Caputo derivative cases can be initial values, function values themselves and integer-order derivatives. Because these data can be measured in practice and have well understood physical meanings, commonly we choose Caputo derivative to deal with concrete physical applications.

According to the predictor-corrector approach for the numerical solution of Caputo fractional differential equations ${ }^{[35,36]}$. The function $f$ is assumed to be a unique solution exists on an interval $[0, \mathrm{~T}]$, and this technique is working on a uniform grid $\left\{t_{\mathrm{n}}=n h: n\right.$ $=0,1, \ldots, N\}$ with some integer $N$ and $h=T / N$. The predicted value is

$$
x_{h}^{p}\left(t_{n+1}\right)=x_{k=0}^{[1] 1} x_{0}^{(k)} \frac{t_{n+1}^{k}+\frac{h}{k !}}{(\quad)}{ }_{j=0}^{n}{ }_{j, n+1} f\left(t_{j}, x_{h}\left(t_{j}\right)\right)
$$

where

$$
\beta_{j, n+1}=\frac{h^{\alpha}}{\alpha}\left((n-j+1)^{\alpha}-(n-j)^{\alpha}\right), 1 \leq j \leq n
$$

And the corrector formula is

$$
x_{h}\left(t_{n+1}\right)={ }_{k=0}^{[1]} x_{0}^{(k)} \frac{t_{n+1}^{k}+\frac{h}{k !}}{(+2)} f\left(t_{n+1}, x_{h}^{p}\left(t_{n+1}\right)\right)+\frac{h}{(+2)}{ }_{j=0}^{n} a_{j, n+1} f\left(t_{j}, x_{h}\left(t_{j}\right)\right)
$$

where

$$
a_{j, n+1}=\left\{\begin{array}{l}
n^{\alpha+1}-(n-\alpha)(n+1)^{\alpha}, j=0 \\
(n-j+2)^{\alpha+1}+(n-j)^{\alpha+1}-2(n-j+1)^{\alpha+1}, 1 \leq j \leq n \\
1, j=n+1
\end{array}\right.
$$

Then Equation (13) can be rewritten in form of Caputo fractional differential equations. With the use of identified parameters in Table 2, the numerical solutions can be taken as predictions of this constitutive model.

$$
\begin{gathered}
(t)=G_{m}(t)+G_{1} \quad D_{{ }_{v}}(t) \\
D_{v}(t)=G_{0}(t) \quad G_{0}{ }_{v}(t)
\end{gathered}
$$

Figure 5 demonstrates the experimental data and the numerical result of this constitutive model. When sinusoidal loads are applied to MRE samples, the response stress will lag behind the input strain by a loss angle. That is why the relationship 
between the shear stress and the shear strain seems to be approximately elliptical in shape. The slop of the main axis of hysteresis loop shows that the dynamic modulus decreases with the increasing strain amplitude, and increases with the frequency and the magnetic flux density. Consequently, the comparison under varying frequency, strain amplitude and magnetic field indicates that the predictions of this constitutive model describe the hysteresis loop well, and the experimental results support the model predictions.

In Figure 6, the numerical results of this fractional-order nonlinear model and the revised Bouc-Wen model (refered to Appendix $\mathrm{A}^{[31]}$ ) are compared with the experimental results. It can be seen that the resulting stress lags slightly behind the input strain, and the loss angle is deduced to be very small. Because the fractional-order nonlinear model can make predictions closer to the experimental data than the revised Bouc-Wen model, the comparison displays that this fractional-order nonlinear model owns a better efficiency from the prospect of precision accuracy. It can be observed from the Equation (20) that the square of the standard error of estimation is the mean squared error, and for the two models the mean squared errors $S_{\mathrm{e}}^{2}$ are presented in Figure 7. The comparison also supports this fractional-order nonlinear model with a much higher precision accuracy.

\section{Conclusion}

In this work, a fractional-order nonlinear model was proposed and validated for MREs to describe the dynamic mechanical behavior. The model parameters were identified through the experimental data fitting of dynamic modulus, and the numerical solution of this proposed model was obtained with the predictor-corrector approach. Subsequently, the comparisons of the stress-strain hysteresis and the response stress exhibited an excellent agreement between the model predictions and the experimental results, which supports the description of dynamic mechanical behavior in various magnetic fields both in time and frequency domains. Finally, this fractional-order nonlinear model proved to own a better efficiency from the prospect of precision accuracy by comparing with the results of the revised Bouc-Wen model. The identified model parameters in this piece of work can be further expressed as functions of magnetic field and strain amplitude to facilitate the dynamic analysis in vibration control systems. 


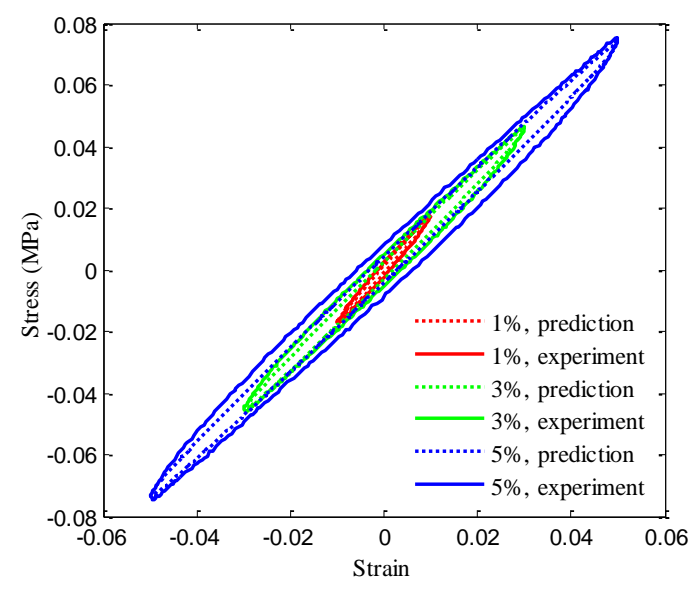

(a)

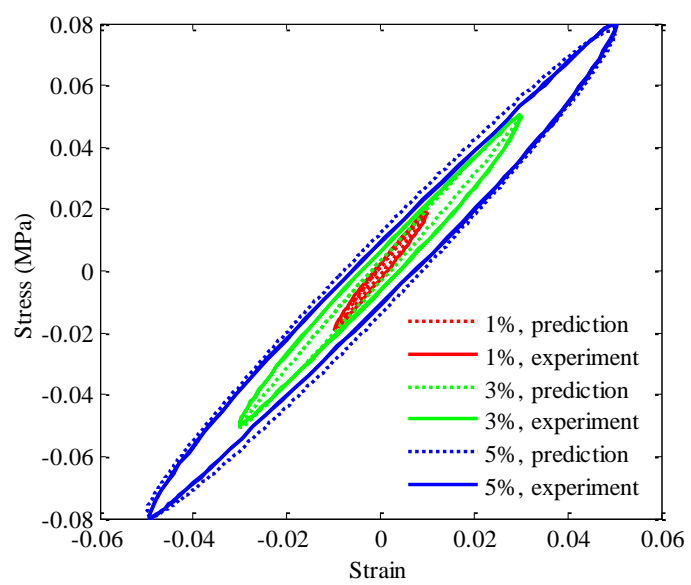

(c)

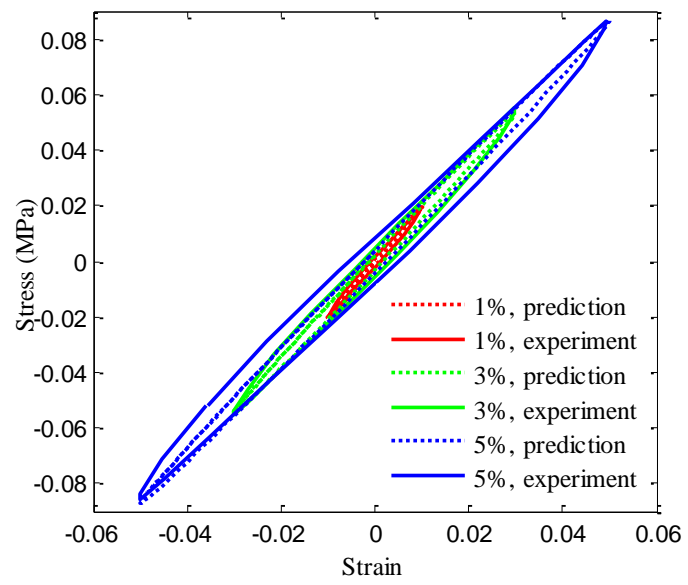

(e)

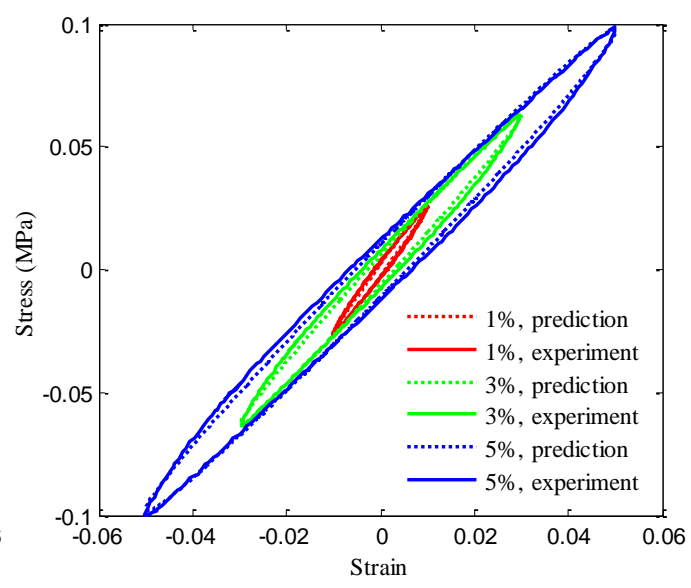

(b)

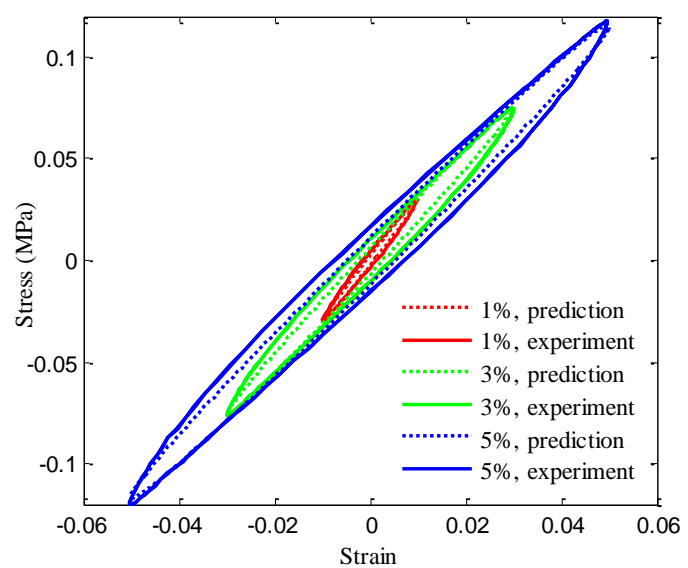

(d)

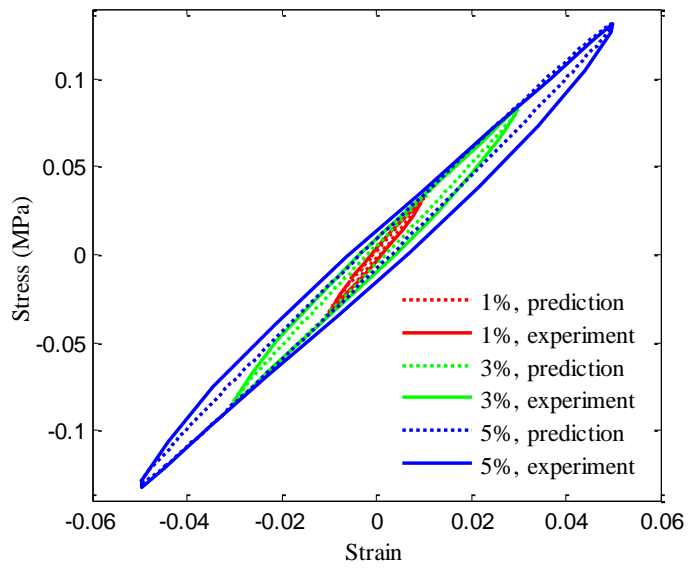

(f)

Figure 5 Comparison between the experimental results and the model predictions of hysteresis loop with $0 \mathrm{mT}$ at (a) $1 \mathrm{~Hz}$, (c) $10 \mathrm{~Hz}$, (e) $50 \mathrm{~Hz}$; and with $500 \mathrm{mT}$ at (b) $1 \mathrm{~Hz}$, (d) $10 \mathrm{~Hz}$, (f) $50 \mathrm{~Hz}$. 


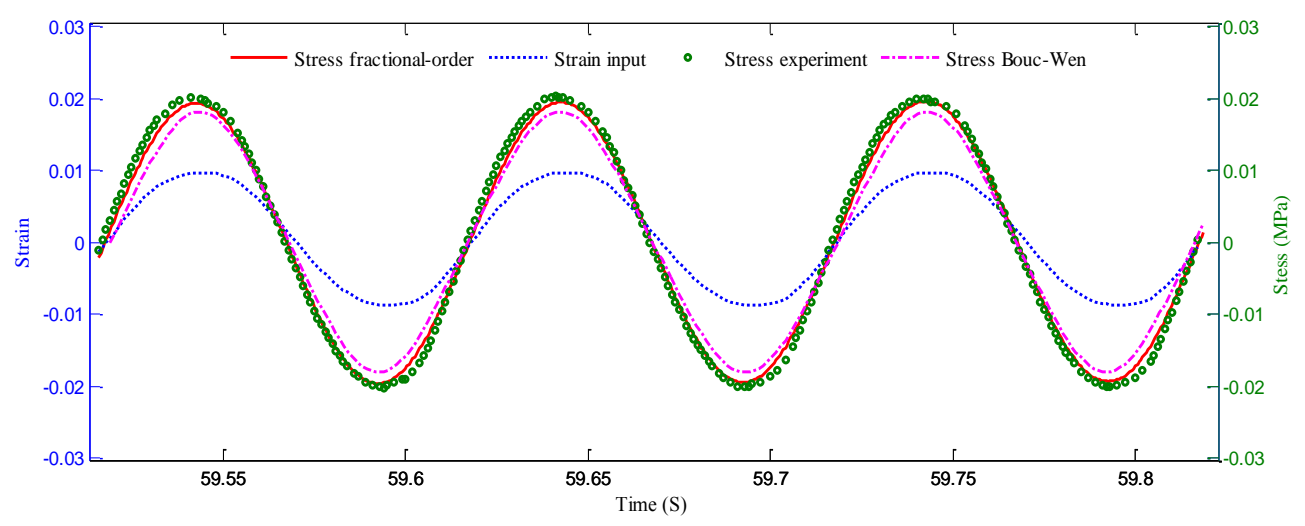

(a)

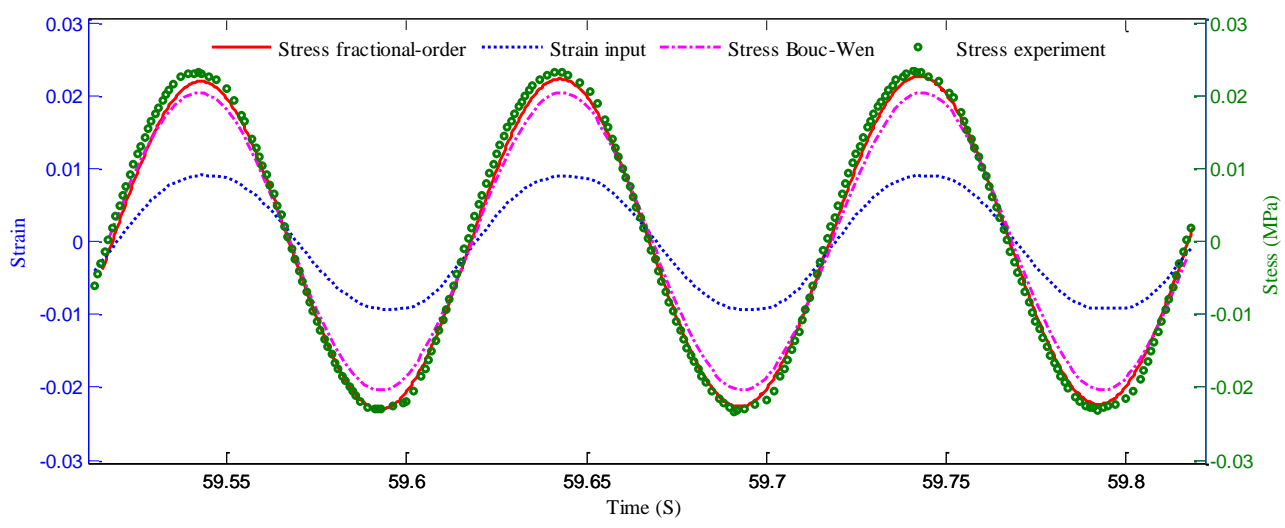

(b)

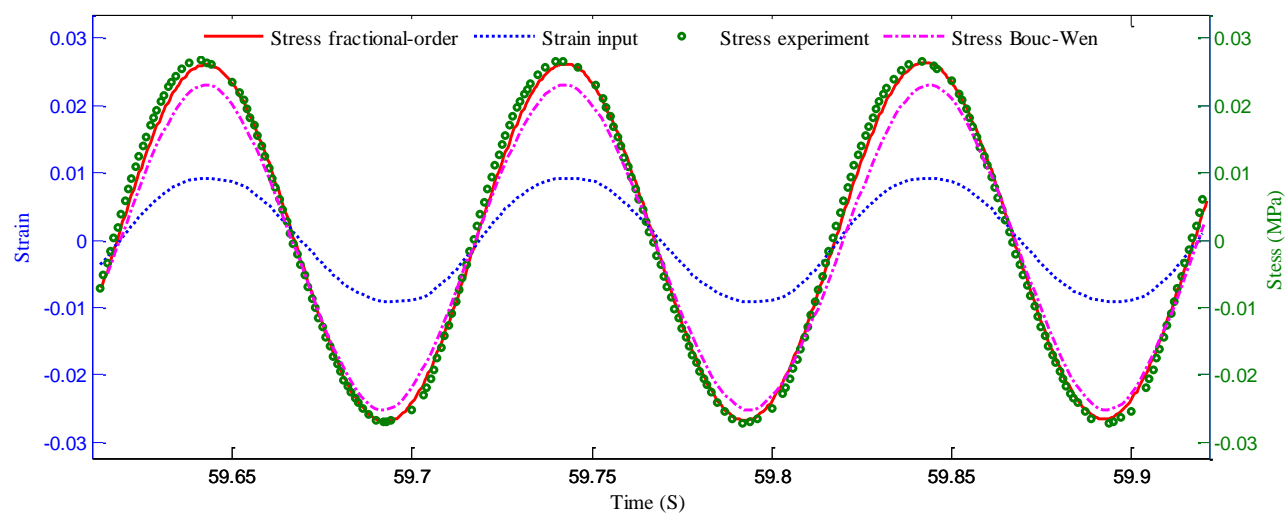

(c)

Figure 6 Comparison of the predicted response stress between the proposed fractional-order model and the revised Bouc-Wen model at $10 \mathrm{~Hz}$ with the strain amplitude of $1 \%$ (a) $0 \mathrm{mT}$ (b) $260 \mathrm{mT}$ (c) 500 $\mathrm{mT}$. 


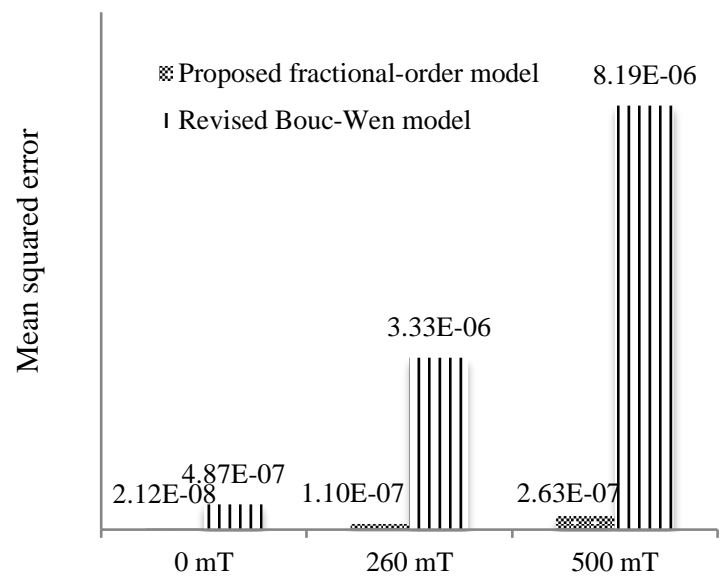

Figure 7 The comparison of $S_{\mathrm{e}}^{2}$ of the proposed fractional-order model and the revised Bouc-Wen model.

\section{Acknowledgement}

This study was funded by National Natural Science Foundation of China (grant numbers 11702213, 11802229 and 11972282) and Natural Science Foundation of Shaanxi Province (grant number 2018JQ1061).

\section{References}

[1] Kaleta J, Królewicz M and Lewandowski D 2011 Magnetomechanical properties of anisotropic and isotropic magnetorheological composites with thermoplastic elastomer matrices Smart Mater. Struct. 20085006

[2] Lu X, Qiao X, Watanabe H, et al. 2011 Mechanical and structural investigation of isotropic and anisotropic thermoplastic magnetorheological elastomer composites based on poly(styrene-b-ethylene-co-butylene-b-styrene) (sebs) Rheol. Acta 51 37-50

[3] Sapouna K, Xiong Y P and Shenoi R A 2017 Dynamic mechanical properties of isotropic /anisotropic silicon magnetorheological elastomer composites Smart Mater. Struct. 26115010 [4] Ibrahim R A 2008 Resent advances in nonlinear passive vibration isolators J. Sound Vib. $314371-452$

[5] Li Y, Li J, Li W and Du H 2014 A state-of-the-art review on magnetorheological elastomer devices Smart Mater. Struct. 23123001

[6] Liu C, Jing X, Daley S and Li F 2015 Recent advances in micro-vibration isolation Mech. Syst. Signal Pr. 56 55-80

[7] Li Y, Li J, Tian T and Li W 2013 A highly adjustable magnetorheological elastomer base isolator for applications of real-time adaptive control Smart Mater. Struct. 22095020

[8] Fu J, Li P, Wang Y, Liao G and Yu, M 2016 Model-free fuzzy control of a magnetorheological elastomer vibration isolation system: analysis and experimental evaluation Smart Mater. Struct. 25035030

[9] Wen Q, Wang Y and Gong X 2017 The magnetic field dependent dynamic properties of magnetorheological elastomers based on hard magnetic particles Smart Mater. Struct. 26 075012 
[10] Xin F L, Bai X X and Qian L J 2017 Principle, modeling and control of a magnetorheological elastomer dynamic vibration absorber for powertrain mount systems of automobiles J. Intell. Mater. Syst. Struct. 28 2239-2254

[11] Qian L J, Xin F L, Bai X X and Wereley N M 2017 State observation based control algorithm for dynamic vibration absorbing systems featuring magnetorheological elastomers: Principle and analysis J. Intell. Mater. Syst. Struct. 28 2539-2556

[12] Nguyen X B, Komatsuzaki T, Iwata Y and Asanuma H 2018 Modeling and semi-active fuzzy control of magnetorheological elastomer-based isolator for seismic response reduction Mech. Syst. Signal Pr. 101 449-466

[13] Boczkowska A and Awietjan S F 2011 Effect of the elastomer stiffness and coupling agents on rheological properties of magnetorheological elastomers WIT Trans. Eng. Sci. 72 263-274

[14] Li R and Sun L Z 2013 Viscoelastic responses of silicone-rubber-based magnetorheological elastomers under compressive and shear loadings J. Eng. Mater. Technol. 135021008

[15] Qiao X, Lu X, Gong X, Yang T, Sun K and Chen X 2015 Effect of carbonyl iron concentration and processing conditions on the structure and properties of the thermoplastic magnetorheological elastomer composites based on poly(styrene-b-ethylene-co-butylene-bstyrene) (SEBS) Polym. Test. 47 51-58

[16] Jolly M R, Carlson J D and Munoz B C 1996 A model of the behavior of magnetorheological materials Smart Mater. Struct. 5 607-614

[17] Davis L C 1999 Model of Magnetorheological Elastomers J. Appl. Phys. 85 3348-3351

[18] Chen L, Gong X L and Li W H 2007 Microstructures and viscoelastic properties of anisotropic magnetorheological elastomers Smart Mater. Struct. 16 2645-2649

[19] Wan Y X, Xiong Y P and Zhang S M 2018 Temperature dependent dynamic mechanical properties of Magnetorheological elastomers: Experiment and modelling Compos. Struct. 202 768-773

[20] Wan Y X, Xiong Y P and Zhang S M 2019 Temperature effect on viscoelastic properties of anisotropic magnetorheological elastomers under compression Smart Mater. Struct. 28 015005

[21] Stolbov O V, Raikher Y L, Stepanov G V, Chertovich A V, Kramarenko E Y and Khokhlov A R 2010 Low-frequency rheology of magnetically controlled elastomers with isotropic structure Polym. Sci. Ser. A 52 1344-1354

[22] Li W H, Zhou Y and Tian T F 2010 Viscoelastic properties of MR elastomers under harmonic loading Rheol. Acta $\mathbf{4 9}$ 733-740

[23] Behrooz M, Wang X J and Gordaninejad F 2014 Modeling of a new semi-active/passive magnetorheological elastomer isolator Smart Mater. Struct. 2345013

[24] Eem S H, Jung H J and Koo J H 2012 Modeling of Magneto-Rheological Elastomers for Harmonic Shear Deformation IEEE T. Magn. 48 3080-3083

[25] Yu G J, Lin X G and Guo F 2017 Modelling and Verification of Relaxation Behavior for Magnetorheological Elastomers with Applied Magnetic Field Key Eng. Mater. 730 527-532

[26] Guo F Du C and Li R 2014 Viscoelastic Parameter Model of Magnetorheological Elastomers Based on Abel Dashpot Adv. Mech. Eng. 6629386

[27] Zhu J T, Xu Z D and Guo Y Q 2012 Magnetoviscoelasticity parametric model of an MR 
elastomer vibration mitigation device Smart Mater. Struct. 21075034

[28] Nadzharyan T A, Sorokin V V, Stepanov G V, Bogolyubov A N and Kramarenko E Y 2016 A fractional calculus approach to model rheological behavior of soft magnetic elastomers Polymer 92 179-188

[29] Nadzharyan T A, Kostrov S A, Stepanov G V and Kramarenko E Y 2018 Fractional rheological models of dynamic mechanical behavior of magnetoactive elastomers in magnetic fields Polymer 142 316-329

[30] Xin F L, Bai X X and Qian L J 2016 Modeling and experimental verification of frequency-, amplitude-, and magnetodependent viscoelasticity of magnetorheological elastomers Smart Mater. Struct. 25105002

[31] Wang Q, Dong X F, Li L Y and Ou J P 2018 Mechanical modeling for magnetorheological elastomer isolators based on constitutive equations and electromagnetic analysis Smart Mater. Struct. 27065017

[32] Norouzi M, Alehashem S M S, Vatandoost H, Ni Y Q and Shahmardan M M 2016 A new approach for modeling of magnetorheological elastomers J. Intell. Mater. Syst. Struct. 271121 1135

[33] Sasso M, Palmieri G and Amodio D 2011 Application of fractional derivative models in linear viscoelastic problems Mech. Time-Depend. Mat. 15 367-387

[34] Yusoff N I M, Chailleux E and Airey G D 2011 A Comparative Study of the Influence of Shift Factor Equations on Master Curve Construction Int. J. Pav. Res. Technol. 4 324-336

[35] Diethelm K, Ford N J and Freed A D 2002 A Predictor-Corrector Approach for the Numerical Solution of Fractional Differential Equations Nonlinear Dynam. 29 3-22

[36] Li C and Deng W 2007 Remarks on fractional derivatives Appl. Math. Comput. 187 777784 


\section{Appendix A}

We are using the revised Bouc-Wen model proposed by Wang et al. ${ }^{[31]}$. The constitutive equations of the revised Bouc-Wen model of the MREs can be expressed by

$$
\begin{aligned}
& \tau=k_{0} \gamma+Z+c_{0} \dot{\gamma}+m_{0} \ddot{\gamma} \\
& \dot{Z}=A \dot{\gamma}-\beta|\dot{\gamma}||z|^{n-1} Z-\gamma_{\mathrm{bW}} \dot{\gamma}|z|^{n}
\end{aligned}
$$

where $\tau$ and $\gamma$ are the shear stress and shear strain, respectively; $A, \beta, \gamma_{\mathrm{bw}}$ and $n$ are the non-dimensional parameters to regulate the shear stress-shear strain hysteresis loop; parameters $c_{0}$ and $k_{0}$ are the coefficients of the Kelvin-Voigt model; and $z$ is the evolutionary variable. All of the parameters are identified with the experimental data using GA.

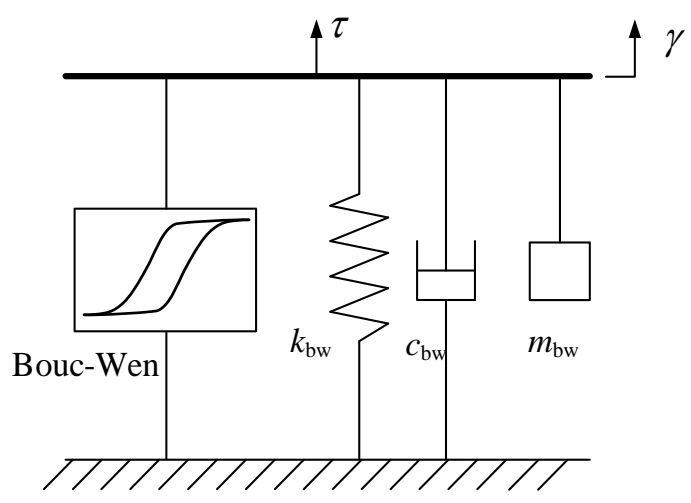

Figure A1. Schematic of the revised Bouc-Wen model. 